\title{
Short- and Long-Term Results of Open Versus Laparoscopic Appendectomy
}

\author{
H. A. Swank • E. J. Eshuis • M. I. van Berge Henegouwen • \\ W. A. Bemelman
}

Published online: 7 April 2011

(C) The Author(s) 2011. This article is published with open access at Springerlink.com

\begin{abstract}
Background Clinical advantages of laparoscopic appendectomy have been shown in numerous trials and reviews. Most of these advantages are small and of limited clinical relevance, while laparoscopic operation costs are reported to be higher. The present study compares short- and longterm results of conventional appendectomy with or without diagnostic laparoscopy (OA), and laparoscopic appendectomy (LA).

Methods All adult patients who underwent appendectomy in our institution from 1995 to 2005 were included retrospectively. Patient data were retrieved from medical records, questionnaires sent by mail, and records of general practitioners. Primary outcome parameters were long-term complications, readmissions, and reinterventions ( $>30$ days postoperatively). Secondary outcome parameters were short-term complications, readmissions, and reinterventions ( $\leq 30$ days postoperatively).

Results A total of 755 patients were included, 545 of whom underwent OA, with the remaining 210 undergoing LA. In the long term there were few complications noted, and there were no significant differences in complications between the two groups. Within 30 days postoperatively, LA was associated with a significantly higher incidence of abdominal abscesses with consequent diagnostic investigations, interventions, and readmissions.
\end{abstract}

H. A. Swank · E. J. Eshuis · M. I. van Berge Henegouwen W. A. Bemelman $(\square)$

Department of Surgery, Academic Medical Centre, University of Amsterdam, 22660, 1100 DD Amsterdam, The Netherlands

e-mail:w.a.bemelman@amc.uva.nl
Conclusions Although laparoscopic appendectomy is known to deliver clinical advantages, it is associated with a higher incidence of abdominal abscesses. Because the procedure is about to become the standard of care, future research must be directed at solving this issue. The expected lower incidence of incisional hernia and small bowel obstruction after laparoscopic appendectomy was not shown in the present study.

\section{Introduction}

Three small incisions, little pain, and quick recovery. The advantages of laparoscopy for acute appendicitis are easily explained to the patient, and its use for the treatment of acute appendicitis has gained great popularity since its introduction [1]. Some surgeons embrace laparoscopic appendectomy, others use the laparoscope as a diagnostic tool and perform conventional appendectomy after diagnostic laparoscopy. However, conventional appendectomy using a McBurney's incision without prior laparoscopy is still performed as well.

The clinical advantages of laparoscopic appendectomy have been shown in numerous trials and two systematic reviews [2, 3]. Most of these advantages are small and of limited clinical relevance.

The present study was designed to compare short- and long-term results of the two recognized treatments of acute appendicitis: conventional appendectomy with or without prior diagnostic laparoscopy (OA), and laparoscopic appendectomy (LA). It was hypothesized that higher complication rates in the short term for laparoscopic appendectomy are compensated in the long term by lower reoperation rates for incisional hernia and adhesions. 


\section{Patients and methods}

All patients over 18 years of age who underwent appendectomy in the Academic Medical Centre, Amsterdam, from 1 January 1995 until 31 December 2005 were included in this retrospective study. Primary outcome parameters were long-term complications, readmissions, and reinterventions ( $>30$ days postoperatively). Secondary outcome parameters were short-term complications, readmissions, and reinterventions ( $<30$ days postoperatively).

Patients were selected from all operative records that were coded "appendectomy." Appendectomies by midline laparotomy (65) or Pfannenstiel incision (2) were excluded, as well as incidental appendectomies (3).

Age, sex, and the pre- and postoperative surgical history of each patient were obtained from the medical files of all patients until 1 June 2009. Perioperative data were retrieved from the operative reports. These data included the type of surgery, age of the patient at the time of operation, experience of the surgeon(s), extent of peritonitis, lavage of the abdomen, and closure of the skin. The type of surgery was recorded according to the intention to treat principle. Appendectomy by McBurney's incision, preceded by planned diagnostic laparoscopy with either one, two, or three trocars, was considered open appendectomy. When a three-trocar attempt was made to mobilize the appendix laparoscopically or to dissect the mesoappendix, the procedure was considered a laparoscopic appendectomy, and a subsequent McBurney's incision was considered a conversion.

Experience of the surgeon(s) was recorded as one consultant, one consultant assisted by a surgical trainee, two surgical trainees, one surgical trainee, or one surgical trainee supervised by a consultant. The extent of peritonitis was recorded as "generalized purulent peritonitis" when the operative report made mention of pus in all four quadrants or all over the abdomen. When limited pus was noted, it was recorded as "local purulent peritonitis." Abdominal cleansing was recorded as "suction only" when only suction of abdominal fluids was performed. "Abdominal lavage" was recorded for either local or diffuse rinsing of the abdomen, "lavage of the wound" was recorded when lavage of the wound was performed but without abdominal lavage. Additional antibiotics were routinely prescribed for five or seven days postoperatively if purulent peritonitis or fecal spill had been documented.

The histological diagnosis was retrieved from the pathology report. A normal appendix lacked signs of inflammation and was characterized by normal vascularization. A flegmonous inflammation was diagnosed based on the finding of granulocytes, fibrosis, fibrin and/or purulent deposits, vascular dilatation, serosal vascular injection, or hemorrhaging. A gangrenous appendix was characterised by tissue destruction, discoloration, (vascular) necrosis, or thrombosis. In a perforated appendix an overt perforation was present.

The record of the hospital stay on either the surgical ward or the intensive care unit was retrieved from the medical files, along with postoperative complications, interventions, and diagnostic investigations.

Recorded diagnostic measures were computed tomography (CT) scanning, ventilation/perfusion (V/Q) scanning, abdominal echography, plain abdominal and thoracic X-ray films, and cultures. To rule out potential postoperative admissions and interventions in other hospitals, a questionnaire with detailed questions about hospital admissions and reoperations after the appendectomy was sent to all living patients with known contact information and residency in the Netherlands.

A reminder was sent to the patients who did not reply to the initial questionnaire. When there was no response, or when the patient had died or emigrated, a questionnaire was sent that patient's general practitioner, who was asked to search the patient's medical history to retrieve any record of hospitalization or treatment related to acute appendicitis, and to look for complaints of incisional hernia and abdominal adhesions.

Statistical analysis was performed with SPSS for Windows, version 17.0 (SPSS Inc. Chicago, IL). The Pearson chi-squared test or Fisher's exact test was used to compare categorical or dichotomous variables between the groups. The median test was used for comparing the medians of age, operative time, and hospital stay. The nonparametric Mann-Whitney $U$-test was used to test for differences between quantitative variables. Logistic regression was used to perform multivariate analysis with binary outcome.

\section{Results}

\section{Study profile}

A total of 755 patients were included in the present study. Some $72 \%$ of the patients underwent conventional appendectomy (OA), and in the other $28 \%$ the operation was performed laparoscopically (LA). A diagnostic laparoscopy preceded $32 \%$ of the conventional appendectomies, and $7 \%$ of the laparoscopic appendectomies required conversion to a McBurney's incision. Some of the patients had died $(n=34)$ or emigrated $(n=46)$, or the contact information was missing or false $(n=50)$. A questionnaire was sent to the remaining 625 patients, which was replied by $46 \%$. After they received a reminder, another 87 patients responded. Patient information was obtained by the general practitioner for another 246 patients. Overall, a response rate of $83 \%$ was achieved (Fig. 1). 


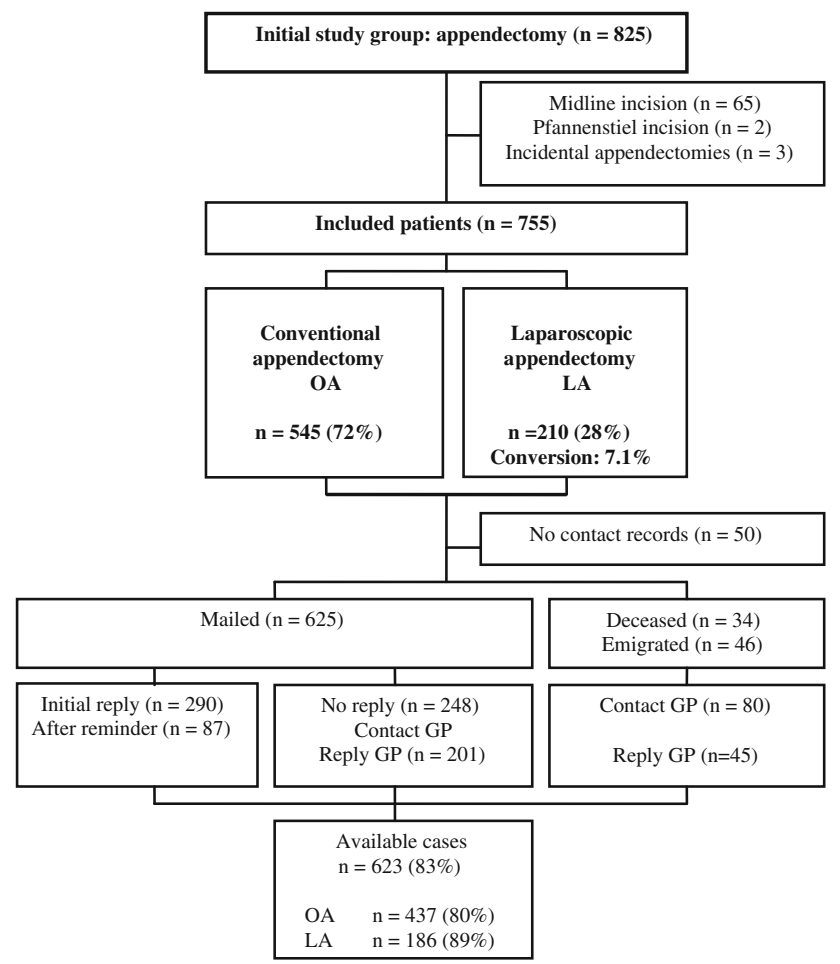

Fig. 1 Study profile

Patient characteristics

Demographics for the two groups are shown in Table 1. The groups had an identical median age (34 years). The OA group consisted of more men $(63 \%)$, while the LA group was dominated by women $(63 \%)$. Prior abdominal surgery, unrelated to appendicitis, was found in $16.3 \%$ (OA) and $16.2 \%$ (LA) of the patients (NS). There was no significant difference in the mean follow-up of the two groups: 9.0 years $(\mathrm{OA})$ vs. 8.7 years $(\mathrm{LA} ; p=0.321)$.

Operative characteristics

Details of the different operative procedures are presented in Table 2. The median operative time in the OA group was significantly shorter than in the laparoscopic group (55 vs. $70 \mathrm{~min} ; p<0.001)$. The histopathological diagnoses were distributed equally between the groups, as was the

Table 1 Baseline data

\begin{tabular}{lllll}
\hline & OA \\
$n=545$ & $\begin{array}{l}\text { LA } \\
n=210\end{array}$ & $\begin{array}{l}\text { Conversion } \\
\mathrm{n}=17\end{array}$ & $p$ Value \\
\hline Sex & & & & \\
$\quad$ Male & $63 \%$ & $37 \%$ & 4 & 0.000 \\
$\quad \begin{array}{l}\text { Female } \\
\begin{array}{c}\text { Age, years, } \\
\text { median (range) }\end{array}\end{array}$ & $37 \%$ & $63 \%$ & 13 & \\
\hline
\end{tabular}

Table 2 Operative data

\begin{tabular}{|c|c|c|c|c|}
\hline & $\begin{array}{l}\text { OA } \\
n=545\end{array}$ & $\begin{array}{l}\text { LA } \\
n=210\end{array}$ & $\begin{array}{l}\text { Conversion } \\
\mathrm{n}=17\end{array}$ & $p$ Value \\
\hline Diagnostic laparoscopy & $31.6 \%$ & & & \\
\hline 1 trocar & $9.7 \%$ & & & \\
\hline 2 trocars & $19.8 \%$ & & & \\
\hline 3 trocars & $2.0 \%$ & & & \\
\hline \multicolumn{5}{|l|}{ Operative time (min) } \\
\hline Median & 55 & 70 & 89 & 0.000 \\
\hline Mean & 59 & 71 & 90 & 0.000 \\
\hline Experience & & & & 0.000 \\
\hline Consultant/trainee & $3.5 \%$ & $14.3 \%$ & 2 & \\
\hline Trainee/consultant & $9.9 \%$ & $20.0 \%$ & 4 & \\
\hline Consultant & $3.7 \%$ & $7.6 \%$ & 2 & \\
\hline Trainee/trainee & $43.9 \%$ & $27.1 \%$ & 5 & \\
\hline Trainee & $39.1 \%$ & $31.0 \%$ & 4 & \\
\hline Appendix & & & & 0.829 \\
\hline Normal & $7.5 \%$ & $5.2 \%$ & 1 & \\
\hline Flegmonous & $45.5 \%$ & $45.2 \%$ & 4 & \\
\hline Gangrenous & $10.1 \%$ & $10.0 \%$ & 0 & \\
\hline Perforated & $19.4 \%$ & $21.4 \%$ & 10 & \\
\hline Not specified & $17.4 \%$ & $18.1 \%$ & 2 & \\
\hline Purulent peritonitis & & & & 0.007 \\
\hline Local & $27.5 \%$ & $17.6 \%$ & 4 & \\
\hline Generalized & $5.5 \%$ & $9.0 \%$ & 5 & \\
\hline Wound & & & & 0.000 \\
\hline Lavage & $3.7 \%$ & $0.5 \%$ & 6 & \\
\hline Abdomen & $25.0 \%$ & $41.9 \%$ & 10 & \\
\hline Suction only & $1.8 \%$ & $1.9 \%$ & 1 & \\
\hline
\end{tabular}

negative appendectomy rate. Open appendectomy was mainly performed by trainees, with a consultant present in $17 \%$ of the cases. Surgical expertise was significantly higher in the LA group: a consultant was present in $42 \%$ of laparoscopic appendectomies. Purulent peritonitis was more common in the OA group (33\%) than in the LA group $(27 \% ; p<0.05)$. Lavage of the abdomen or the wound was performed in $29 \%$ (OA) and $42 \%$ (LA) of the patients, most often (64\%) upon finding a purulent peritonitis.

The skin was left open in 19\% (OA) and 4\% (LA) of the patients $(p<0.001)$, in $73 \%$ because of purulent peritonitis. In three out of nine of the LA cases, the McBurney's incision was left open after conversion.

\section{Short-term results}

In the short term, results varied between the two groups (Table 3). Open appendectomy led to a longer median postoperative hospital stay (4 days) than in the LA group ( 3 days; $p=0.093$ ). The same distinction accounted for the longer total hospital stay ( 4 vs. 3 days; $p=0.066$ ). 
Table 3 Short-term results: $<30$ days after initial surgery

\begin{tabular}{lllll}
\hline & OA $(\%)$ & LA $(\%)$ & Conversion & $p$ Value \\
& $n=545$ & $n=210$ & $\mathrm{n}=17$ & \\
\hline Abdominal abscess & 1.5 & 6.2 & 2 & 0.001 \\
Percutaneous drainage & 1.1 & 5.7 & 2 & 0.001 \\
Readmission & 2.2 & 6.7 & 2 & 0.004 \\
Ileus: conservative & 2.4 & 3.4 & 0 & 0.308 \\
Wound infection or & 2.6 & 1.4 & 1 & 0.259 \\
$\quad$ abscess & & & & \\
Relaparotomy & 1.1 & 1.9 & 2 & 0.292 \\
Urinary tract infection & 0.4 & 1.4 & 1 & 0.135 \\
Cardiac disease & 0.7 & 1.4 & 1 & 0.303 \\
Wound dehiscence & 0.0 & 0.5 & 0 & 0.278 \\
Pneumonia & 0.4 & 0.5 & 0 & 0.624 \\
Mortality & 0.2 & 0.0 & 0 & 0.722 \\
\hline
\end{tabular}

However, OA was associated with significantly fewer abdominal abscesses $(2 \%)$ than LA $(6 \% ; p=0.001)$. The lower rate of abscess was associated with a lower rate of percutaneous drainage ( 1 vs. $6 \% ; p=0.001)$. Patients in the OA group received fewer postoperative diagnostic investigations: fewer patients needed CT scanning (3\% $[\mathrm{OA}]$ versus $9 \%[\mathrm{LA}] ; p=0.001)$, abdominal echography (6 vs. $12 \% ; p<0.01)$ and V/Q scanning (0 vs. $2 \%$; $p<0.05)$. There were no significant differences for the number of plain X-ray films and the number of cultures obtained.

Patients in the OA group had a lower risk of readmission ( 2 vs. $7 \% ; p<0.005$ ). The rates of all other complications were not significantly different, although one patient in the OA group died.

In the LA group 15 patients required conversion to conventional appendectomy (7\%). Most of these patients were women $(76 \% ; p=0.183)$, often with a history of abdominal surgery (35 vs. $15 \% ; p<0.05$ ) and a higher median age (40 vs. 33 years; $p=0.150)$. Generalized purulent peritonitis was the only risk factor $(p=0.001)$ for conversion in a logistic regression model (OR 8.04 CI 2.46-26.24). Prior abdominal surgery and the histopathological diagnosis were not found to be significant factors.

Converted patients had a higher risk of relaparotomy (13 vs. $1.0 \%$ in the non-converted group; $p<0.05$ ) and incisional hernia (13 vs. $0.0 \% ; p=0.005$ ), which was not caused by the longer duration of surgery.

Twenty-three patients developed abdominal abscesses. Most of these patients were men $(65 \%, \mathrm{NS})$, and their median age was 7 years older than that of patients who did not experience an abscess (41 vs. 34 years; $p=0.099$ ). In a multivariate analysis, significant risk factors for abscesses were the duration of surgery (OR 1.01 CI 1.00-1.03) and purulent peritonitis $(p=0.03)$. Both local (OR $2.8 \mathrm{CI}$
1.1-7.1) and generalized purulent peritonitis (OR 4.1 CI 1.2-14.0) were significant risk factors, whereas the histopathological diagnosis was not.

Abdominal lavage was not included in the analysis, because this factor was highly associated with the finding of purulent peritonitis. In $67 \%$ of the patients who underwent abdominal lavage, local or generalized pus was present.

\section{Long-term results}

There was no long-term mortality (Table 4). There were three abdominal abscesses in the OA group. The incidence of incisional hernia was similarly low: $0.7 \%(\mathrm{OA})$ vs. $1.0 \%$ (LA; NS). There were no trocar site hernias: all cases of incisional hernia were found at the McBurney's incision site, and all were surgically corrected in our institution. The readmission rate was $1.1 \%(\mathrm{OA})$ vs. $1.4 \%$ (LA; NS). There was no significant difference for reoperation rates. No cases of small bowel obstruction were found.

\section{Discussion}

From this retrospective study evaluating short- and longterm results of appendectomy, we conclude that laparoscopic appendectomy did not have clear advantages with respect to reduced short- and long-term morbidity.

Laparoscopic appendectomy was associated with a higher rate of intra-abdominal abscesses and adverse events in the group of converted patients, giving rise to significantly more diagnostic and therapeutic interventions. The overall incidence of long-term morbidity and reoperation for incisional hernia repair or small bowel obstruction was low and not significantly different between the groups.

Both open and laparoscopic appendectomy were performed in our institution. The open approach was predominantly used in men, while the laparoscopic group was dominated by women. This difference was caused by the hospital protocol that recommended starting with a

Table 4 Long-term results: $>30$ days after initial surgery

\begin{tabular}{lllll}
\hline & OA (\%) & LA (\%) & Conversion & $p$ Value \\
& $n=545$ & $n=210$ & $\mathrm{n}=17$ & \\
\hline Readmission & 1.1 & 1.4 & 1 & 0.478 \\
Abdominal abscess & 0.6 & 0.0 & 0 & 0.376 \\
Percutaneous drainage & 0.4 & 0.0 & 0 & 0.521 \\
Incisional hernia & 0.7 & 1.0 & 2 & 0.532 \\
$\quad$ Incisional hernia & 0.7 & 1.0 & 2 & 0.532 \\
$\quad$ correction & & & & 0.521 \\
Relaparotomy & 0.4 & 0.0 & 0 & \\
\hline
\end{tabular}


laparoscopic approach in women to exclude gynecological pathology. In men, both approaches were supported and the type of approach depended on the available laparoscopic expertise and time in the out of office hours situation.

Although the laparoscopic approach gained popularity over the years, the open approach was most commonly in our study. While the attending consultant has preferred the laparoscopic approach, the operating trainees might not have felt familiar with the laparoscopic procedure in the first years after the introduction of LA; they may have opted for the fastest procedure they could do unsupervised. Over the years, the laparoscopic procedure has been performed more frequently (Fig. 2), and it is now the preferred treatment.

Midline laparotomy has been used several times in the course of this study, with poor clinical outcome. A suspicion of acute appendicitis is nowadays unlikely to be an indication for midline laparotomy. The group of patients that was eligible for this procedure was older and tended to have perforated appendicitis with associated generalized peritonitis. Therefore we have excluded these cases.

The operative time for both OA and LA was, respectively, 15 and 12 min longer than found by Sauerland et al.'s meta-analysis [2]. This can be caused by operations performed in the teaching setting, as was the case in at least 14 and $34 \%$ of the patients, respectively. Limited surgical or laparoscopic expertise might have played a role too, yet we were not able to stratify for this variable.

Considering the short-term outcomes, significantly different parameters were postoperative stay and short-term abdominal abscesses, readmissions, diagnostics, and reinterventions. The postoperative stay was one day shorter after LA, which is according to Sauerland et al. [2]. However, the difference in our study was small and therefore difficult to interpret. Although the discharge date may have a significant meaning both for the patient as for the financial system, it is influenced by the surgeon's and patient's expectations as well as by social and personal factors.

The most important outcome of the present study is the high rate of abdominal abscess in the LA group, significantly associated with the finding of a purulent peritonitis and a long duration of surgery. Male gender and a higher age were correlated, but not significantly. All these findings are in accordance with the finding the large series of Fleming et al., who have shown that patients with a high wound class, systematic illness, sepsis, male gender, a history of smoking, and operative time of more than $60 \mathrm{~min}$ have a significantly greater risk of abdominal abscess after the laparoscopic procedure [4].

Conversion was necessary in $7 \%$ of the patients that were intentionally treated laparoscopically. The only significant risk factor for conversion was the presence of a generalized purulent peritonitis. Although patients with previous abdominal surgery had a higher risk of conversion, this was not significant when corrected for sex, age, and surgical experience. The converted patients were at risk for relaparotomy and incisional hernia, and this finding was independent of the duration of surgery.

In literature, there is no evidence that laparoscopic appendectomy is contraindicated for patients with either complicated appendicitis or a history of abdominal surgery $[5,6]$. Further on, preoperative selection of complicated cases is challenging, as CT findings of appendiceal abscess and extraluminal gas are associated with a high specificity but a low sensitivity in relation to perforated appendicitis [7].

With long-term morbidity as our primary endpoint, we expected the incidences of incisional hernia and small bowel obstruction to be higher in the open appendectomy group. The incidence of reoperation for incisional hernias after open appendectomy was $1.1 \%$ in our study. Data are
Fig. 2 Included patients per year

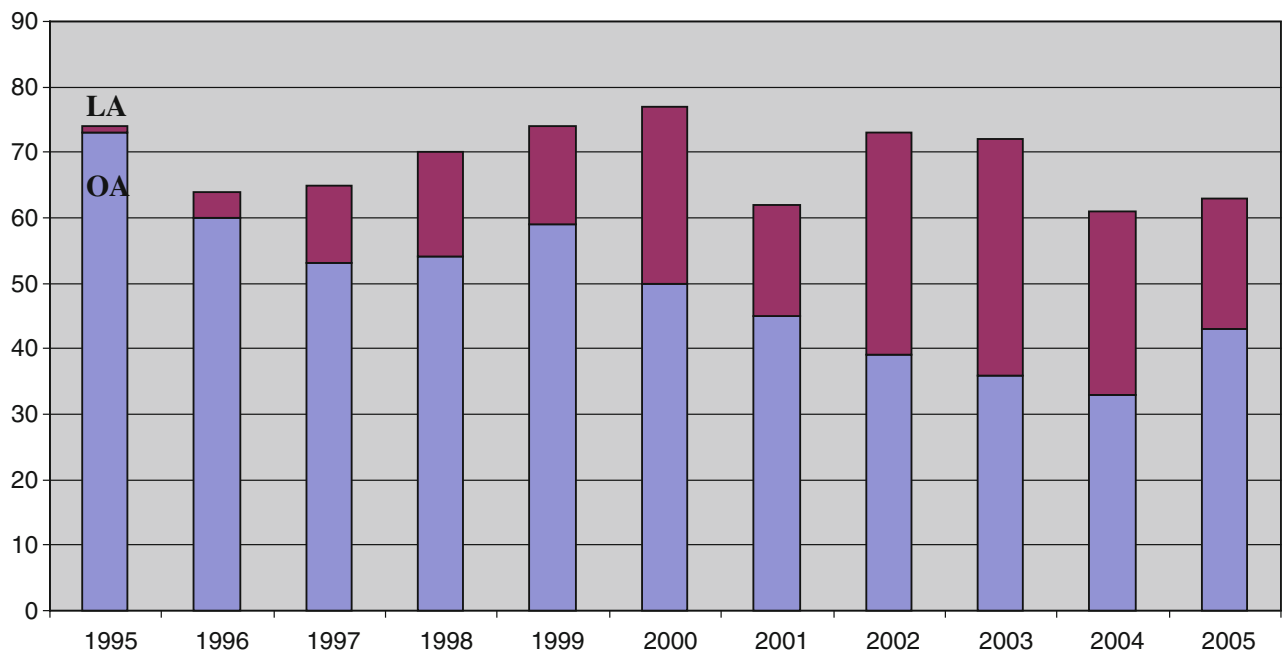


lacking on the incisional hernia rate in the group of patients who did not request reoperation: it is possible that the rate in fact is higher, but that it exists in a group that never asked for a correction.

Incisional hernia was observed after laparoscopic appendectomy as well, however exclusively in the patients that were converted. Therefore no significant difference was found in our study between the open and laparoscopic group. The available literature shows incidences of $0.12-0.7 \%$ incisional hernia after open appendectomy [8-10] With increasing laparoscopic expertise, one might expect the number of converted operations to be reduced, thereby lowering the number of incisional hernias in the LA group.

We did not encounter patients with small bowel obstruction, where prospective studies reported incidences of $1.0-2.8 \%$ [11-13]. It is suggested that small bowel obstruction can present many years after surgery. In the large retrospective series from Andersson et al., the prevalence of bowel obstruction after appendectomy increased from $0.63 \%$ after 1 year, to $0.97 \%$ after 10 years, to $1.30 \%$ after 30 years of follow-up [11]. Therefore our follow-up with a minimum of three years has been too short to reliably retrieve all cases of small bowel obstruction.

The Achilles' heel of laparoscopic appendectomy remains the increased incidence of intra-abdominal abscesses. Although we do not know the specific cause of this problem, certain risk factors have been identified: male gender, long operative duration, and particularly purulent peritonitis.

Despite the concerns about LA for complicated appendicitis, it is not to be expected that LA will be abandoned because of abdominal abscess. Apart from the cosmetic expectations of the patient, laparoscopic appendectomy delivers limited clinical benefits in the short term and a possible lower risk of incisional hernia in the long term. We expect it to be standard treatment for all cases of acute appendicitis in the future. The problem of abdominal abscess therefore needs to resolved, and further research should be directed on this topic.

One direction for future research is the use of the Endo Stapler. Two systematic reviews have advised the use of this device instead of endoloops to close the appendiceal stump in laparoscopic appendectomy, although no high quality studies could be included. Staplers most likely reduce fecal spill and provide a more secure stump closure $[14,15]$. Properly designed studies must be conducted addressing the cost-effectiveness of this technique.
Open Access This article is distributed under the terms of the Creative Commons Attribution Noncommercial License which permits any noncommercial use, distribution, and reproduction in any medium, provided the original author(s) and source are credited.

\section{References}

1. Bauwens K, Schwenk W, Bohm B et al (1998) Recovery and duration of work disability after laparoscopic and conventional appendectomy. A prospective randomized study. Chirurg 69: 541-545

2. Sauerland S, Jaschinski T, Neugebauer EA (2010) Laparoscopic versus open surgery for suspected appendicitis. Cochrane Database Syst Rev 10:CD001546

3. Sauerland S, Lefering R, Holthausen U et al (1998) Laparoscopic vs conventional appendectomy - a meta-analysis of randomised controlled trials. Langenbecks Arch Surg 383:289-295

4. Fleming FJ, Kim MJ, Messing S et al (2010) Balancing the risk of postoperative surgical infections. Ann Surg 252:895-900

5. Wullstein C, Barkhausen S, Gross E (2001) Results of laparoscopic vs. conventional appendectomy in complicated appendicitis. Dis Colon Rectum 44:1700-1705

6. Wu JM, Chen KH, Tseng LM et al (2007) Impact of previous abdominal surgery on laparoscopic appendectomy for acute appendicitis. Surg Endosc 21:570-573

7. Bixby SD, Lucey BC, Soto JA et al (2006) Perforated versus nonperforated acute appendicitis: accuracy of multidetector CT detection. Radiology 241:780-786

8. Beltran MA, Cruces KS (2008) Incisional hernia after McBurney incision: retrospective case-control study of risk factors and surgical treatment. World J Surg 32:596-601

9. Konstantakos AK, Zollinger RM Jr (2000) Repair of McBurney incisional hernias after open appendectomy. Curr Surg 57:79-80

10. Tingstedt B, Johansson J, Nehez L et al (2004) Late abdominal complaints after appendectomy-readmissions during long-term follow-up. Dig Surg 21:23-27

11. Andersson RE (2001) Small bowel obstruction after appendicectomy. Br J Surg 88:1387-1391

12. Leung TT, Dixon E, Gill M et al (2009) Bowel obstruction following appendectomy: what is the true incidence? Ann Surg 250:51-53

13. Khairy GA, Afzal MF, Murshid KR et al (2005) Post appendectomy small bowel obstruction. Saudi Med J 26:1058-1060

14. Sajid MS, Rimple J, Cheek E et al (2009) Use of endo-GIA versus endo-loop for securing the appendicular stump in laparoscopic appendicectomy: a systematic review. Surg Laparosc Endosc Percutan Tech 19:11-15

15. Kazemier G, In't Hof KH, Saad S et al (2006) Securing the appendiceal stump in laparoscopic appendectomy: evidence for routine stapling? Surg Endosc 20:1473-1476 